\title{
The Effect of Virtual Reality Training on Unilateral Spatial Neglect in Stroke Patients
}

\author{
Yong Mi Kim, M.D., Min Ho Chun, M.D., Gi Jeong Yun, M.D., Young Jin Song, O.T., Han Eun Young, M.D. \\ Department of Rehabilitation Medicine, Asan Medical Center, University of Ulsan College of Medicine, Seoul 138-736, \\ ${ }^{1}$ Jeju National University Hospital, Jeju 690-756, Korea
}

\begin{abstract}
Objective To investigate the effect of virtual reality training on unilateral spatial neglect in stroke patients. Method Twenty-four stroke patients (14 males and 10 females, mean age=64.7) who had unilateral spatial neglect as a result of right hemisphere stroke were recruited. All patients were randomly assigned to either the virtual reality (VR) group ( $n=12)$ or the control group $(n=12)$. The VR group received VR training, which stimulated the left side of their bodies. The control group received conventional neglect therapy such as visual scanning training. Both groups received therapy for 30 minutes a day, five days per week for three weeks. Outcome measurements included star cancellation test, line bisection test, Catherine Bergego scale (CBS), and the Korean version of modified Barthel index (K-MBI). These measurements were taken before and after treatment.

Results There were no significant differences in the baseline characteristics and initial values between the two groups. The changes in star cancellation test results and CBS in the VR group were significantly higher than those of the control group after treatment. The changes in line bisection test score and the K-MBI in the VR group were not statistically significant.

Conclusion This study suggests that virtual reality training may be a beneficial therapeutic technique on unilateral spatial neglect in stroke patients.
\end{abstract}

Key Words Neglect, Stroke, Hemiplegia, Virtual reality

\section{INTRODUCTION}

Unilateral spatial neglect is a syndrome in which patients act as if whole regions of space contralateral to their damaged brain lesions do not exist although their visual field is normal. This unilateral spatial neglect generally results from right hemisphere damage and may

Received February 19, 2010; Accepted November 23, 2010

Corresponding author: Min Ho Chun

Department of Rehabilitation Medicine, Asan Medical Center, University of Ulsan College of Medicine, 388-1, Pungnab 2-dong, Songpa-gu, Seoul 138-736, Korea

Tel: +82-2-3010-3800, Fax: +82-2-3010-6964, E-mail: mhchun@amc. seoul.kr

Copyright $\odot 2011$ by Korean Academy of Rehabilitation Medicine also results from damaged parietal lobe, frontal lobe, thalamus, or basal ganglia. ${ }^{1-3}$ Patients with unilateral spatial neglect are not aware of any damage on the paretic side, so they may bump into an object or face ambulatory risks or high risks of falling. These patients are limited in their ability to carry out activities of daily living, such as getting dressed and practicing personal hygiene. ${ }^{4,5}$ Previous studies have found that unilateral spatial neglect independently affected trunk balance in stroke patients. ${ }^{6}$ Other studies also found that unilateral spatial neglect affected trunk balance six to twelve weeks after stroke occurrence. ${ }^{7}$ In addition, unilateral spatial neglect hampers effects of rehabilitation treatments and slows down functional recovery. The most common 
treatment for unilateral spatial neglect is visual tracking. Additionally, other treatment methods have been tested. ${ }^{8}$ In particular, prism therapy was reported to maintain therapeutic effects compared to other therapies. ${ }^{9}$ Beis et al. ${ }^{10}$ reported that blindfolding the damaged eye improved unilateral spatial neglect by inducing eyeballs to move toward the paretic side. Schindler et al. ${ }^{11}$ reported that unilateral spatial neglect could be temporally improved by inducing eyeballs to move toward the paretic side with stimulation of the vestibular organ. However, these studies have their own limitations because they were not conducted on patients.

A virtual reality (VR) is a computer-generated environment in which people can feel and interact with neighboring situations in three dimensions. It minimizes the gap between a lab environment and an actual situation, and the therapy can be applied using the interface regardless of physical impairments. ${ }^{12}$ Due to these beneficial aspects, VR therapy is widely used as treatment and is an assessment tool for brain-injured patients. In particular, it was reported that the therapy was effective in improving cognitive functions such as memory and concentration. ${ }^{13,14}$ Recently, a small number of techniques based on VR have been suggested for the treatment of unilateral spatial neglect. ${ }^{15-17}$ However, few studies have shown the effect of VR therapy. Furthermore, few studies have examined the effects of VR therapy compared to other treatment methods through randomized controlled trials.

Thus, this study was designed to determine if VR therapy would improve unilateral spatial neglect in comparison to conventional rehabilitation programs, with the goal of it becoming the recommended therapy in unilateral spatial neglect rehabilitation.

\section{MATERIALS AND METHODS}

\section{Subjects}

The study was conducted on stroke patients who were diagnosed as unilateral spatial neglect through the line bisection test or star cancellation test out of those admitted to the Department of Rehabilitation from September 2008 to October 2009. Patients who had the following problems were excluded: 1) Patients with severe cognitive impairment or aphasia who could not understand the instructions given by therapists, 2)
Table 1. Baseline Characteristics of Subjects

\begin{tabular}{lcc}
\hline & Control group & VR group \\
\hline Number (n) & 12 & 12 \\
Gender (Male : Female) & $5: 7$ & $9: 3$ \\
Age (years) & $67.2 \pm 13.9$ & $62.3 \pm 10.2$ \\
Time post stroke (days) & $25.5 \pm 18.5$ & $22.8 \pm 7.6$ \\
Right MCA infarction (n) & 12 & 12 \\
Right dominant (n) & 12 & 12 \\
K-MMSE & $22.5 \pm 4.2$ & $22.0 \pm 4.3$ \\
\hline
\end{tabular}

VR: Virtual reality, K-MMSE: Korean Mini-Mental State Examination, MCA: Middle cerebral artery

${ }^{*} \mathrm{p}<0.05$

patients who had so severely damaged sitting balance that they could not sit down on a chair with a back and armrests, 3) patients with problems in cervical movement due to orthopedic impairment, and 4) patients who could not recognize objects on a computer screen due to severely damaged eyesight. A total of 24 patients were selected and randomly assigned to either the control group or the VR group. The control group was 67.2 years old on average while the VR group was 62.3 years old. The mean elapsed times from the outbreak of stroke to the start of rehabilitation were 25.5 and 22.8 days, respectively. Their dominant limb was right, and they had right cerebral artery infarction. No significant differences in age, elapsed time, and Korean Mini-Mental State Examination existed between the control group and the VR group (Table 1).

\section{Method}

Physical therapy, occupational therapy, and cognitive therapy of the same intensity and time were applied to all patients. In addition, two occupational therapists conducted treatment for unilateral spatial neglect. One therapist conducted conventional rehabilitation programs for the control group, such as visual tracking, reading and writing, drawing and copying, and puzzles, while the other conducted the virtual reality treatment on the VR group. Such treatments were applied for thirty minutes a day, once a day and five days a week for three weeks. The VR system (IREX system ${ }^{\circledR}$, Vivid group, Toronto, Canada) consists of a monitor, a video camera, computer-recognizing gloves, and virtual objects (Fig. 1-A). The video camera recognizes movements 

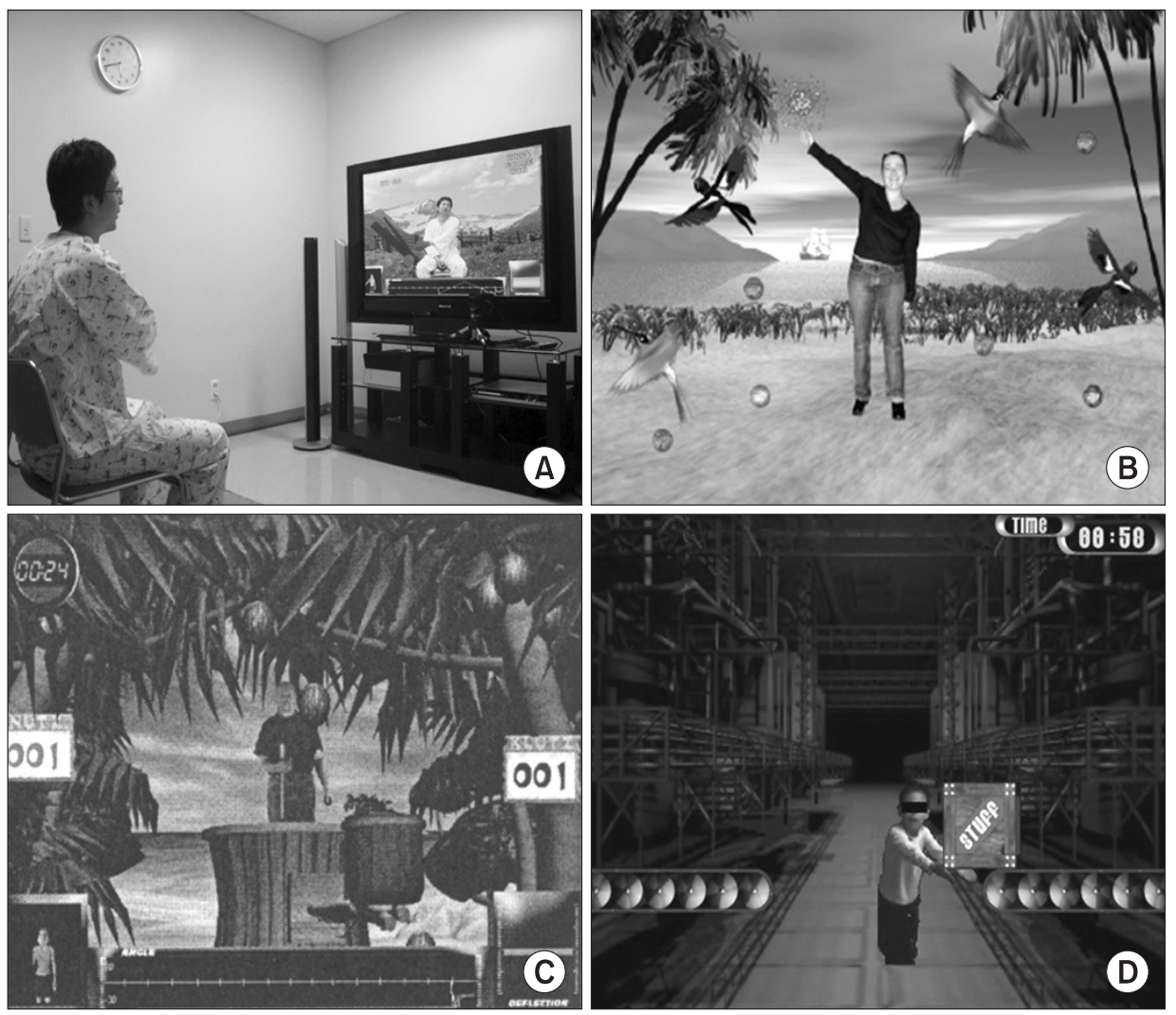

Fig. 1. (A) Virtual reality experimental set up (IREX system ${ }^{\circledR}$, Vivid group, Toronto, Canada), (B) Birdball, (C) Coconut, (D) Conveyer.

or position of a patient and transfers him or her to the virtual space. The computer gloves read responses of a patient to the virtual reality. All patients sat in a wheelchair because they could not be trained while standing. The VR training is composed of three programs at a time. "Bird and Ball" is a program in which a patient touches a flying ball with his or her hand and turns it into a bird (Fig. 1-B). The second program, "Coconut," is a program in which a patient catches a coconut falling from a tree while moving around (Fig. 1-C). In the third program, "Container," a patient moves a box carried in a container to the opposite side (Fig. 1-D). In the three programs, patients were told to use non-paretic right hands against the left stimulation. When patients were not able to respond to the stimuli, the therapist encouraged them to respond. Calibration was made for three minutes before the start of the training. There were 150 seconds of resting between programs and 30 seconds between treatments. Each session was repeated three times.

\section{Assessment method}

The two groups took the star cancellation test, line bisection test, and Catherine Bergego Scale (CBS) 24 hours before and after the three-week treatment to assess the severity of unilateral spatial neglect. Those tests were conducted by a therapist who was not involved in the treatment and did not know the state of the patients. However, CBS was filled out by guardians under the supervision of the therapist. In the line bisection test, the deviation between the point that a patient thought a center of the line and the actual center point was divided by a half of the line length and the mean was obtained by summing up the percentage figures. The mean figure tended to get large in patients with severe unilateral spatial neglect. ${ }^{18}$ Missing lines were excluded from the calculation. In the star cancellation test, the neglected stars on the left were counted; the figure got large in patients with severe neglect. ${ }^{19} \mathrm{CBS}$ is a questionnaire. Guardians were allowed to fill out the questionnaire. The larger the score, the more severe the unilateral spatial neglect. ${ }^{20}$ The Korean version of modified Barthel Index (K-MBI) was used to assess the daily activity performance.

The collected data were statistically processed with SPSS 14.0 version, and the level of significance was set 
Table 2. Clinical Parameters before and after Treatment

\begin{tabular}{lccccc}
\hline & \multicolumn{2}{c}{ Control group } & & \multicolumn{2}{c}{ VR group } \\
\cline { 2 - 3 } \cline { 5 - 6 } & Before treatment & After treatment & & Before treatment & After treatment \\
\hline Star cancellation test & $11.4 \pm 8.2$ & $6.9 \pm 7.8^{*}$ & & $15.3 \pm 9.3$ & $8.2 \pm 8.3^{*}$ \\
Line bisection test & $10.8 \pm 9.9$ & $5.9 \pm 8.7^{*}$ & & $24.9 \pm 22.2$ & $18.9 \pm 22.6^{*}$ \\
CBS & $17.9 \pm 7.1$ & $13.2 \pm 7.6^{*}$ & & $20.1 \pm 7.5$ & $11.0 \pm 5.7^{*}$ \\
K-MBI & $34.4 \pm 22.1$ & $44.9 \pm 21.8^{*}$ & & $28.5 \pm 15.6$ & $47.9 \pm 15.1^{*}$ \\
\hline
\end{tabular}

VR: Virtual reality, CBS: Catherine Bergego scale, K-MBI: Korean version of modified Barthel index ${ }^{*} \mathrm{p}<0.05$

at $\mathrm{p}<0.05$. General characteristics were analyzed with technical statistics to find out the level of detail items. A Wilcoxon Test was used to compare the severity of neglect for both groups before and after treatment, and a MannWhitney U Test was employed to compare differences in both groups before and after treatment.

\section{RESULTS}

Seven subjects in the control group and three subjects in the VR group accurately performed the line bisection test and were included in the analysis. All the patients conducted the other tests accurately. The two groups did not show any significant difference in the star cancellation test, line bisection test, CBS, and K-MBI before treatment. After rehabilitation treatment, both groups showed significant improvements in the star cancellation test, line bisection test, CBS, and K-MBI ( $\mathrm{p}<$ 0.05) (Table 2).

The VR group showed a higher increase in the star cancellation test and CBS scores after treatment than the control group $(\mathrm{p}<0.05)$, but both groups did not show any difference in the line bisection test and K-MBI before and after treatment (Table 3).

\section{DISCUSSION}

Virtual reality (VR) provides a realistic environment and is easy to control. It is also safe and cost-effective. Therefore, VR technology is more widely employed for medical purposes. In particular, it was reported that a wider application of the technology brought treatment effects to post-traumatic stress disorder, panic disorder, or attention deficit hyperactivity disorder. ${ }^{21-23}$ It is also widely used in cognitive rehabilitation in the field of brain diseases. ${ }^{14}$ The application range of VR technology
Table 3. Comparison of Difference after 3 week Treatment in Each Group

\begin{tabular}{lcc}
\hline & Control group & VR group \\
\hline Star cancellation test & $4.1 \pm 7.2$ & $8.7 \pm 7.9^{*}$ \\
Line bisection test & $4.9 \pm 2.2$ & $5.7 \pm 4.6$ \\
CBS & $4.6 \pm 3.8$ & $9.1 \pm 6.3^{*}$ \\
K-MBI & $15.5 \pm 9.0$ & $19.5 \pm 9.4$ \\
\hline
\end{tabular}

VR: Virtual reality, CBS: Catherine Bergego scale, K-MBI: Korean version of modified Barthel index ${ }^{*} \mathrm{p}<0.05$

is extending to include activities of daily living and sports activities. ${ }^{24,25}$ However, it is still an early stage to apply VR therapy to unilateral spatial neglect patients. ${ }^{26}$ The scope of application for unilateral spatial neglect includes assessment and rehabilitation. Kim et al. ${ }^{12}$ demonstrated that unilateral spatial neglect could be effectively assessed using measurement of deviation degree and concentration span, utilizing a virtual reality program in which patients wearing head-mounted devices were instructed to find the center with cervical movements. Buxbaum et al. ${ }^{15}$ assessed unilateral spatial neglect by deviation degree when patients simulated driving a wheelchair in the virtual reality. Various rehabilitation therapies for unilateral spatial neglect are suggested, but there are few studies that compare these therapies to conventional rehabilitation programs. Additionally, few studies have examined the effectiveness of such treatments by comparison to a control group while conducting sustained treatment for a certain period of time. Castiello et al. ${ }^{16}$ reported the effect of VR application when they arranged patients to grab a real object placed right to the center and provided the re-arranged virtual reality where objects were placed left to the center. This study, however, was conducted only on six patients, and 
the treatment programs were applied three times for one day to compare results before and after treatment so it had its own limitations in proving sustained effects. Katz et al. ${ }^{27}$ reported that the control group showed greater improvements in conventional rehabilitation programs, virtual reality assessment, and actual street crossing than the VR group when the VR group underwent street crossing in the virtual reality and the control group performed visual tracking on a computer.

This study also observed the virtual reality program was more effective for unilateral spatial neglect than conventional rehabilitation programs through the three-week treatment. The assessments included the line bisection test, star cancellation test, and a CBS questionnaire. This study argued that the virtual reality program was very useful for assessing unilateral spatial neglect. However, the fact that the virtual reality group received training in VR and thus had experience with it when the control group had not suggests that the learning effect might have influenced the assessment. As such, the severity of neglect was not assessed with virtual reality in this study. As a result, no significant improvement in the line bisection test was observed. This seems to be attributed to the exclusion of patients who did not perform the test properly, meaning only a few patients were included in the assessment. Both the VR and control groups did not show significant improvement in activities of daily living, as other factors, such as motor performance of four limbs, cognition, and coordination work together for daily activities, not to mention unilateral spatial neglect.

This study had its own limitations. The subjects were randomly assigned to either the VR group or control group, and both groups showed no difference in initial studies. However, they had large standard deviations, so it might have worked to overwhelm a statistical difference in severity of initial neglect. The reason for greater improvement in the VR group is that the degree of improvement in the range with a wide standard deviation is greater than that in the range with narrow standard deviation. Thus, it could be that the difference in the improvement degree between two groups resulted from the difference in severity of unilateral spatial neglect. Therefore, future studies may require assessments for differences in severity of unilateral spatial neglect. In the case of CBS, guardians were allowed to fill in the questionnaire. Unlike other double-blind assessments, the guardians knew about the treatment of their patients, which means this study was not completely double-blind. Though the treatment programs were applied for three weeks and follow-up was not available, it was meaningful that the virtual reality therapy was suggested as one of the rehabilitation methods for unilateral spatial neglect even if many therapeutic methods were not proposed. Subtypes of unilateral spatial neglect were not assessed in this study, but it is presumed that the star cancellation test might bring about some improvement in not only the motor area but also the peripersonal area. It was difficult to identify an area in unilateral spatial neglect that was improved by virtual reality therapy because CBS included personal, extrapersonal, peripersonal, and motor areas. Future studies should extend the treatment period or the tracking/observation period and take subtypes of neglect into consideration.

The following hypotheses for the cause of unilateral spatial neglect have been suggested: 1) decreased attention and arousal on the contralesional side of space, 2) a damaged right hemisphere dominant attention-shift mechanism, and 3) a deficit in an internal representation of the contralesional side of space ${ }^{17}$ Castiello and Glover et al. ${ }^{16,28}$ applied virtual reality therapy to improve remapping of space in the brain, capitalizing on the deficit in an internal representation of the contralesional side of space among the hypotheses and reported that only patients who had non-damaged inferior parietal and superior parietal lobes showed improvements with virtual reality therapy (remapping of space in the brain). It was subsequently presumed that this area played an important role in improving unilateral spatial neglect. In this study, improvement in decreased attention and arousal seemed to lead to improvement of neglect. Compared to conventional rehabilitation therapies, virtual reality therapy can easily stimulate interest and participation because it gives immediate feedback to incorrect responses in a three-dimensional space, which keeps patients in an arousal state for a prolonged time. Due to this advantage, virtual reality therapy was reported to be useful for improvement of attention in cognitive rehabilitation therapy. Such effects are thought to have positive influences on improvement of attention and arousal compared to conventional rehabilitation therapies. 
Despite the importance of neglect influence on recovery of stroke and daily living activities, the reality is that few known effective therapies are currently in existence. Two of these, prism therapy and visual tracking, are known for their beneficial effects. Prism therapy has low compliance of patients because it is hard to apply. This study reported the effect of using the virtual reality program that stimulates the contralesional side of space. The therapy seems to be easily applied to patients who have decreased cognitive function after stroke or to gerontic patients because it can be easily understood and has no major treatment difficulties.

\section{CONCLUSION}

This study observed that the VR group showed a significant improvement in neglect compared to the control group when the virtual reality program was applied to patients with neglect caused by right hemisphere cerebral infarction. Thus, virtual reality therapy must be considered a potential rehabilitation program for patients with unilateral spatial neglect.

\section{REFERENCES}

1. Damasio AR, Damasio H, Chui HC. Neglect following damage to frontal lobe or basal ganglia. Neuropsychologia 1980; 18: 123-132

2. Heilman KM, Valenstein E. Frontal lobe neglect in man. Neurology 1998; 50: 1202-1207

3. Watson RT, Valenstein E, Heilman KM. Thalamic neglect. Possible role of the medial thalamus and nucleus reticularis in behavior. Arch Neurol 1981; 38: 501-506

4. Chen Sea MJ, Henderson A, Cermak SA. Patterns of visual spatial inattention and their functional significance in stroke patients. Arch Phys Med Rehabil 1993; 74: 355-360

5. Denes G, Semenza C, Stoppa E, Lis A. Unilateral spatial neglect and recovery from hemiplegia: a followup study. Brain 1982; 105: 543-552

6. van Nes IJ, van der Linden S, Hendricks HT, van Kuijk AA, Rulkens M, Verhagen WI, Geurts AC. Is visuospatial hemineglect really a determinant of postural control following stroke? An acute-phase study. Neurorehabil Neural Repair 2009; 23: 609-614
7. van Nes IJ, van Kessel ME, Schils F, Fasotti L, Geurts AC, Kwakkel G. Is visuospatial hemineglect longitudinally associated with postural imbalance in the postacute phase of stroke? Neurorehabil Neural Repair 2009; 23: 819-824

8. Tsirlin I, Dupierrix E, Chokron S, Coquillart S, Ohlmann T. Uses of virtual reality for diagnosis, rehabilitation and study of unilateral spatial neglect: review and analysis. Cyberpsychol Behav 2009; 12: 175-181

9. Luaute J, Halligan P, Rode G, Jacquin-Courtois S, Boisson D. Prism adaptation first among equals in alleviating left neglect: a review. Restor Neurol Neurosci 2006; 24: 409-418

10. Beis JM, Andre JM, Baumgarten A, Challier B. Eye patching in unilateral spatial neglect: efficacy of two methods. Arch Phys Med Rehabil 1999; 80: 71-76

11. Schindler I, Kerkhoff G, Karnath HO, Keller I, Goldenberg G. Neck muscle vibration induces lasting recovery in spatial neglect. J Neurol Neurosurg Psychiatry 2002; 73: 412-419

12. Kim DY, Lee JH, Park CI, Kim YW, Chang WH, Kim IY, Kim SI, Chon J, Chang HJ. Assessment of post-stroke unilateral spatial neglect using 3-dimensional virtual reality program. J Korean Acad Rehab Med 2005; 29: 1-8

13. Klinger E, Bouchard S, Legeron P, Roy S, Lauer F, Chemin I, Nugues P. Virtual reality therapy versus cognitive behavior therapy for social phobia: a preliminary controlled study. Cyberpsychol Behav 2005; 8: 76-88

14. Rizzo AA, Buckwalter JG. The status of virtual reality for the cognitive rehabilitation of persons with neurological disorders and acquired brain injury. Stud Health Technol Inform 1997; 39: 22-33

15. Buxbaum LJ, Palermo MA, Mastrogiovanni D, Read MS, Rosenberg-Pitonyak E, Rizzo AA, Coslett HB. Assessment of spatial attention and neglect with a virtual wheelchair navigation task. J Clin Exp Neuropsychol 2008; 30: 650-660

16. Castiello U, Lusher D, Burton C, Glover S, Disler P. Improving left hemispatial neglect using virtual reality. Neurology 2004; 62: 1958-1962

17. Pierce SR, Buxbaum LJ. Treatments of unilateral spatial neglect: a review. Arch Phys Med Rehabil 2002; 83: $256-268$ 
18. Schenkenberg T, Bradford DC, Ajax ET. Line bisection and unilateral visual neglect in patients with neurologic impairment. Neurology 1980; 30: 509-517

19. Halligan PW, Marshall JC, Wade DT. Visuospatial neglect: underlying factors and test sensitivity. Lancet 1989; 2: 908-911

20. Azouvi P, Olivier S, de Montety G, Samuel C, LouisDreyfus A, Tesio L. Behavioral assessment of unilateral spatial neglect: study of the psychometric properties of the Catherine Bergego Scale. Arch Phys Med Rehabil 2003; 84: 51-57

21. Wallach HS, Bar-Zvi M. Virtual-reality-assisted treatment of flight phobia. Isr J Psychiatry Relat Sci 2007; 44: 29-32

22. Pollak Y, Weiss PL, Rizzo AA, Weizer M, Shriki L, Shalev RS, Gross-Tsur V. The utility of a continuous performance test embedded in virtual reality in measuring ADHD-related deficits. J Dev Behav Pediatr 2009; 30: 2-6

23. Difede J, Hoffman HG. Virtual reality exposure therapy for World Trade Center Post-traumatic Stress Disorder: a case report. Cyberpsychol Behav 2002; 5:
529-535

24. Lotan M, Yalon-Chamovitz S, Weiss PL. Improving physical fitness of individuals with intellectual and developmental disability through a virtual reality intervention program. Res Dev Disabil 2009; 30: 229239

25. Rose FD, Brooks BM, Rizzo AA. Virtual reality in brain damage rehabilitation: review. Cyberpsychol Behav 2005; 8: 241-271

26. Koenig ST, Crucian GP, Dalrymple-Alford JC, Dunser A. Virtual reality rehabilitation of spatial abilities after brain damage. Stud Health Technol Inform 2009; 144: 105-107

27. Katz N, Ring H, Naveh Y, Kizony R, Feintuch U, Weiss PL. Interactive virtual environment training for safe street crossing of right hemisphere stroke patients with unilateral spatial neglect. Disabil Rehabil 2005; 27: 1235-1243

28. Glover S, Castiello U. Recovering space in unilateral spatial neglect: a neurological dissociation revealed by virtual reality. J Cogn Neurosci 2006; 18: 833-843 